Running head: MINDFULNESS IN PSYCHIATRIC DISORDERS

\title{
Acceptance and present-moment awareness in psychiatric disorders: Is mindfulness mood
} dependent?

\author{
Matilde Elices $^{\text {a,b }}$, Rosa Tejedor ${ }^{\mathrm{c}}$, Juan C. Pascual ${ }^{\mathrm{a}, \mathrm{d}}$, Cristina Carmona ${ }^{\mathrm{a}}$, José Soriano ${ }^{\mathrm{a}, \mathrm{d}}$, \\ Joaquim Soler ${ }^{\text {a,d* }}$
}

${ }^{a}$ Department of Psychiatry. Hospital de la Santa Creu i Sant Pau, Barcelona, Spain. Centro de Investigación Biomédica en Red de Salud Mental. CIBERSAM, Madrid, Spain.

${ }^{\mathrm{b}}$ IMIM-Hospital del Mar Medical Research Institute, Barcelona, Spain.

${ }^{c}$ Grup de Recerca en Salut Mental i Innovació Social (SaMIS). Divisió de Salut Mental. Fundació Althaia, Xarxa Assistencial Universitaria de Manresa, Manresa, Spain.

${ }^{\mathrm{d}}$ Department of Psychiatry and Legal Medicine, Autonomous University of Barcelona, UAB, Barcelona, Spain.

*Correspondence to:

Joaquim Soler

C/Sant Antoni Maria Claret 167

08025

Barcelona, Spain.

E-mail: jsolerri@santpau.cat

\section{Conflict of Interest}

Declarations of interest: none. 
MINDFULNESS IN PYSCHIATRIC DISORDERS

Acceptance and present-moment awareness in psychiatric disorders:

\section{Is mindfulness mood dependent?}

\section{Introduction}

Existing definitions of mindfulness suggests two core dimensions: present-centered awareness and nonjudgmental acceptance (Baer et al., 2004; Bishop et al., 2004; Kabat-Zinn, 1990). The first componentpresent centered awareness - refers to the capacity to pay attention and bring awareness to the present moment. The second component-acceptance - refers to an open, non-judgmental, acceptance-based attitude towards whatever arises in the internal and external fields, regardless of whether this is pleasant or unpleasant (Bishop et al., 2004).

From a trait perspective, mindfulness is a dispositional characteristic that varies naturally from one individual to the next (Baer et al., 2004). As a trait, mindfulness can be explored using a range of self-report instruments. These instruments vary in several aspects, mainly with regard to the number of factors assessed and the specific definition of mindfulness on which they are based. The Mindful Attention Awareness Scale (MAAS; Brown and Ryan, 2003), for example, assesses only a single factor (i.e., the individual's dispositional capacity to be present and conscious in daily life experiences), while the Philadelphia Mindfulness Scale (PHLMS; Cardaciotto et al., 2008) reflects the aforementioned twocomponent model, measuring both attention and acceptance. Other instruments, such as the Five Facet Mindfulness Questionnaire (FFMQ; Baer et al., 2006) and the Kentucky Inventory of Mindfulness Skills (KIMS; Baer et al., 2004), are based on a multidimensional concept of mindfulness and assess up to five different factors.

In the last 30 years, mindfulness-based interventions (MBIs) have been increasingly used in the field of mental health (Khoury et al., 2015). Mindfulness has been integrated as a key aspect of broader behavioral interventions, as for example dialectical behavioral therapy (DBT; Linehan, 1993), acceptance and commitment therapy (ACT; Hayes et al., 1999) or the unified protocol for transdiagnostic treatment of emotional disorders (Barlow et al., 2011). Other programs, as mindfulness-based cognitive therapy (MBCT; Teasdale et al., 2000) or mindfulness-based stress reduction (Kabat-Zinn, 1990) place mindfulness practice at the core of the treatment. Some of these interventions have been developed to target specific psychiatric conditions. For example, DBT was originally developed for borderline personality disorder (BPD; Linehan, 1993), and MBCT was designed to help individuals with recurrent depressive episodes (Teasdale et al., 2000). Other programs, such as MBSR, were not developed to treat a 


\section{MINDFULNESS IN PYSCHIATRIC DISORDERS}

specific psychiatric condition but rather to offer a treatment that can be applied to heterogeneous populations suffering from a wide range of mental and physical conditions (Khoury et al., 2013).

Most MBIs were developed to enhance the two components of mindfulness-present-centeredawareness and acceptance - both of which are believed to play a key role in the effectiveness of these interventions (Lindsay and Creswell, 2017). However, recent research has suggested that acceptancerelated capacities play an important role in psychopathology. In recent study by Kotsou, Leys and Fossion (2018), acceptance was a better predictor of psychopathology than other variables, including presentcentered awareness, emotion regulation, and mindfulness. In BPD, research has shown a greater effect of mindfulness training on acceptance-skills compared to attentional skills (Elices et al., 2016; Krantz et al., 2018). Several studies that have used self-report measures have found an inverse association between trait mindfulness and psychiatric conditions, such as BPD (Baer et al., 2004; Elices et al., 2015; Wupperman et al., 2008), eating disorders (ED; Butryn et al., 2013; Lavender et al., 2011), substance abuse (Shorey et al., 2013), and depressive and anxiety symptoms (Arch and Craske, 2010). Interestingly, some studies have found that not all mindfulness capacities are equally affected in individuals with mental disorders. For example, in a study comparing a heterogeneous clinical sample to a non-clinical sample, Tejedor et al., (2014) found significant differences between the clinical and non-clinical samples in the acceptance component of the PHLMS, but no between-group differences in the awareness facet. Similarly, Kohls et al. (2009) found that the negative relationship between mindfulness and anxiety/depression was due primarily to the acceptance factor rather than the awareness component of mindfulness. Similarly, there is evidence suggesting that the severity of BPD symptoms varies according to the individual's acceptancerelated capacities (Eisenlohr-Moul et al., 2016).

Considered as a whole, the findings from the aforementioned studies suggest the presence of deficits in mindfulness skills in clinical populations, especially acceptance. However, these studies understate an interesting issue related to the association between mindfulness-related capacities and psychiatric disorders: although these different clinical groups present certain specific symptoms or clinical characteristics, they also share others. For example, the comorbidity between substance abuse, BPD, or ED and depressive symptoms is common (Rohde et al., 1991). Depressive symptoms appear to affect the attentional domains (Peckham et al., 2010), and therefore an individual's capacity for presentmoment awareness is likely to be impaired when depressive symptoms are high. In fact, MBIs developed for treating recurrent major depressive disorder, such as MBCT, discourage mindfulness training during 
MINDFULNESS IN PYSCHIATRIC DISORDERS

acute phases of the disorder, but rather prefer to conduct mindfulness training between depressive episodes, when attentional capacities are at their best (Teasdale et al., 1995; Teasdale et al., 2000). Nevertheless, to our knowledge, no studies have yet been examined the relationship between presentcentered awareness, acceptance, and depressive symptoms in psychiatric populations. The fact that depressive symptoms are commonly associated with mental disorders such as substance abuse, BPD, and ED (Rohde et al., 1991) raises the question of whether such symptoms could account for mindfulness deficits, regardless of the specific clinical diagnosis. Most of the available studies have compared clinical samples with community samples and thus little is known about the differences and similarities in the mindfulness capacities of individuals with different mental disorders. Moreover, the extent to which clinical samples are characterized by a broad and nonspecific deficit in mindfulness capacities remains unclear. In this context, a better understanding of these relationships might have a relevant impact on the use of MBIs in specific clinical populations, thus helping clinicians to better tailor these interventions. Based on the aforementioned evidence, it seems that at least some psychiatric disorders might benefit more from interventions aimed at enhancing acceptance-related capacities rather than attention-related skills. In addition, a better understanding of the relationship between present-centered awareness, acceptance, and depressive symptoms might be valuable to more accurately assess mindfulness skills in different clinical groups.

The present study had two main aims. First, to investigate the differences and similarities in the mindfulness capacities (i.e., present-centered awareness and acceptance) of individuals with a range of different mental disorders. The second aim was to explore whether depressive symptoms account for differences in mindfulness capacities regardless of the specific clinical diagnosis. Therefore, we explored two different dimensions of mindfulness (acceptance and awareness) among individuals who met clinical criteria for one of the following four disorders: cocaine dependence (CD), ED, BPD, or major depressive disorder in remission (MDDr). A non-clinical community sample was also included as a control group. We hypothesized that these groups (both clinical and non-clinical) would present significant differences in awareness and/or acceptance scores and that depressive symptoms would account for differences in mindfulness capacities regardless of the specific clinical group.

\section{Method}

\subsection{Participants}


MINDFULNESS IN PYSCHIATRIC DISORDERS

The clinical groups were recruited from the psychiatric units at two general hospitals in Catalonia, Spain. Adult patients (18-60 years of age) who met DSM-IV (APA, 2000) criteria for any of the following disorders were invited to participate: $\mathrm{CD}$, ED (i.e., bulimia nervosa, anorexia nervosa, or eating disorder not otherwise specified), MDDr, or BPD.

The non-clinical (NC) group was recruited from nonmedical staff working at the two aforementioned hospitals. Other NC participants included the relatives and acquaintances of the staff members, and independent sources in the community. Participation was offered if they 1) presented no current or past psychiatric disorders according DSM-IV criteria (APA, 2000); 2) scored below 6 on the McLean Screening Instrument for Borderline Personality Disorder (MSI-BPD; Soler et al., 2016; Zanarini et al., 2003); 3) were not taking psychopharmacological treatment; and 4) 18-60 years of age. Participants in both groups (clinical and non-clinical) were excluded if they: 1) fulfilled DSM-IV criteria for a current major depressive episode, 2) had prior experience with mindfulness or other meditative practices; and/or

3) were under current psychological treatment of any kind.

Diagnoses were based on clinical impression using DSM-IV criteria (APA; 2000) and were confirmed using the respective modules of the Structured Clinical Interview for DSM-IV Axis I disorders (SCID-I; First et al., 1999). Only patients in the BPD group were assessed with the Structured Clinical Interview for Axis II disorders (SCID-II; Gibbon and Spitzer, 1997). The MSI-BPD (Soler et al., 2016; Zanarini et al., 2003) was used to rule out the presence of BPD symptoms in the other participants.

From a total of 310 eligible participants, 246 individuals took part in the study (20 individuals declined participation and 44 did not meet the inclusion criteria). The final clinical sample consisted of 162 individuals; of these, 43 had a diagnosis of cocaine dependence, while the remaining participants met clinical criteria for $\operatorname{ED}(n=43), \operatorname{MDDr}(n=39)$, or BPD $(n=45)$. The non-clinical (NC) sample was comprised of 76 individuals.

\section{INSERT TABLE 1 HERE}

\subsection{Procedure}

As part of the routine procedures of mental health services at our hospitals, new patients undergo three initial diagnostic interviews designed to obtain information about the patient's previous medical history and to establish the diagnosis. Participants deemed eligible to participate in the study were invited to join at the end of the diagnostic phase. Those who agreed to participate completed self-report questionnaires at the clinician's office. Participants pertaining to the NC sample were screened briefly by telephone and 
MINDFULNESS IN PYSCHIATRIC DISORDERS

those who agreed were asked to participate in an interview at the hospital where inclusion/exclusion criteria were confirmed and the participants completed the paper and pencil questionnaires. All participants provided written informed consent. The study was reviewed and approved by the ethics committee of the Hospital de la Santa Creu i Sant Pau of Barcelona and performed in accordance with the ethical standards outlined in the Declaration of Helsinki. Participation was voluntary and unremunerated.

\subsection{Measures}

2.2.1 DSM-IV diagnoses. The clinical diagnoses were established on the basis of clinical judgment and were confirmed using the SCID-I (First et al., 1999) or SCID-II (Gibbon and Spitzer, 1997) modules as appropriate. The Spanish version of the MSI-BPD (Soler et al., 2016; Zanarini et al., 2003) was used to rule out the presence of BPD symptoms in the non-BPD sample. The MSI-BPD is a 10-item (dichotomous: yes or no) screening measure for borderline personality symptoms according to DSM-IVTR criteria. Example items include: "Have you deliberately hurt yourself physically (e.g., punched yourself, cut yourself, burned yourself)?" and "Have you been extremely moody?" Participants were asked to answer each item with respect to the past week. The Spanish version of the MSI-BPD (Soler et al., 2016) has shown good psychometric properties, including adequate internal consistency $(\mathrm{KR}-20=$ 0.87), sensitivity (0.71), and specificity (0.68). The Cronbach's alpha in our sample was 0.89 .

\subsubsection{Depressive symptoms. Centre for Epidemiologic Studies Depression Scale (CES-D; Radloff,}

1977; Soler et al., 1997). The CES-D is a 20-item, self-report scale that evaluates the presence of depressive symptoms during the past week. Items cover six areas of symptoms: depressed mood, psychomotor retardation, loss of appetite, sleep disturbance, feelings of guilt/worthlessness and hopelessness. For each item, four possible responses are possible: 0 (rarely, less than a day), 1 (some of the time, 1-2 days), 2 (occasionally, 3-4 days), and 3 (most of the time, 5-7 days). Higher scores (range: 0 to 60) indicate greater symptom severity. The Spanish version (Soler et al., 1997) showed high internal consistency $($ Cronbach's alpha $=0.90)$ and high correlation with the other measures of depression. The Cronbach's alpha in the present study was 0.89 .

\subsubsection{Present-centered awareness. The Mindfulness Attention Awareness Scale (MAAS; Brown and}

Ryan, 2003; Soler et al., 2012) was used to assesses the level of dispositional awareness and attention to the present experience. The scale consists of 15 items rated on a six-point-scale (ranging from 1 [almost always] to 6 [almost never]). Sample items include: "I rush through activities without being really attentive to them" and "I find it difficult to stay focused on what's happening in the present." The Spanish 
MINDFULNESS IN PYSCHIATRIC DISORDERS

version of the MAAS has shown adequate psychometric properties, including internal consistency as well as convergent and discriminant validity (Soler et al., 2012). The Cronbach's alpha in our study was: 0.91 . In addition, the Philadelphia Mindfulness Scale (PHLMS; Cardaciotto et al., 2008; Tejedor et al., 2014) was also used to assess present moment awareness. Sample items of the awareness subscale include: "When talking with other people, I am aware of their facial and body expressions". Items are rated on a five-point-scale $(1=$ never, 5 = very often) based on how frequently these experiences occurred over the previous week. In the this study, the Cronbach's alpha for the present-centered awareness subscale was 0.78 .

2.2.4 Acceptance. To assess the individual's acceptance capacity, the acceptance subscale of the PHLMS was also used (Cardaciotto et al., 2008; Tejedor et al., 2014). Acceptance items refer to the extent to which the individual experiences internal and external events with a non-judgmental stance. A sample item of the acceptance sub-scale is: "I tell myself that I shouldn't feel sad'. On the acceptance subscale, the Cronbach's alpha was 0.85 .

\subsection{Data analyses}

All data were screened for skewness and kurtosis in order to test assumptions of normality (Tabachnick and Fidell, 2000). Between-group differences in socio-demographic and clinical characteristics were analyzed using the chi-square test for categorical variables (or Fisher's exact test if frequencies were $<5$ ) and one-way analysis of variance (ANOVA) for continuous variables. Pearson correlations were calculated to explore the relationship between present-centered awareness and depressive symptoms, and between acceptance and depressive symptoms. To evaluate between-group differences in presentcentered awareness and acceptance, we conducted a set of exploratory analyses: three separate ANOVAs were performed (without using any covariates). If any significant effect of group was found, then post hoc analyses were carried out. Given that several significant between-group differences were found for some sociodemographic variables, additional ANCOVAs were performed to explore the single effects of these variables on present-centered awareness and acceptance. In these ANCOVAs, MAAS, PHLMSawareness or PHLMS-acceptance scores were entered as the dependent variable, while group, sex, and education level were entered as factors, and age and CES-D as covariates. The final models (ANCOVAs) were calculated using only the variables that were significant in the preliminary analyses. If the main effect of group was significant, post hoc test were carried out to explore between-groups differences. Effect sizes of the group differences are reported by partial eta squared, with values up to 0.01 considered 
MINDFULNESS IN PYSCHIATRIC DISORDERS

small, 0.06 considered moderate, and values $\geq 0.14$ considered large (Cohen, 1988). All analyses were conducted using the IBM-SPSS Statistics Program for Windows, version 25 (IBM Corp, 2017). Statistical significance was determined by two-tailed $p<.05$.

\section{Results}

\subsection{Characteristics of the sample}

The sociodemographic profile of the sample was diverse (see Table 1). Significant between-group differences were found for all sociodemographic variables including gender, age, educational level, employment status, and marital status. The majority of the sample was composed of women, except for the CD group in which most participants were males. The mean age was significantly higher in the MDDr group compared to the other clinical groups. The lowest and highest educational levels were observed, respectively, in the CD group (73.2\% with less than a high school education) and the NC sample (84.\% university graduates).A main effect of group was found $[F(4,237)=58.73, p<.001, \eta 2 p=.48)$ for the between-group differences in depressive symptoms. As expected, post hoc analyses indicate that the NC group scored significantly lower than the other groups $(p<.001)$. Among clinical groups, the CD and ED groups scored significantly lower $(p<.001)$ on depressive symptoms than those in the other clinical groups.

\section{INSERT TABLE 1 HERE}

\subsection{Correlations between present-centered awareness, acceptance and depressive symptoms}

Pearson's correlations were calculated to explore the relationship between MAAS and PHLMS scores with depressive symptoms. CES-D scores correlated significantly and inversely with MAAS scores $(r=-$ $.55 ; p<.001)$, with PHLMS-acceptance $(r=-.46 ; p<.001)$ and PHLMS-awareness $(r=-.21, p<.001)$.

\subsection{Between group differences in present-centered awareness}

Preliminary analyses. An ANOVA analysis with MAAS scores as the dependent variable and group as the between-subjects factor revealed a significant main effect of group $[F(4,229)=10.09, p$

$\left.<.001, \eta^{2} \mathrm{p}=.15\right]$. Post hoc analyses showed significant differences between the NC group versus the ED $(p=.004), \mathrm{BPD}(p<.001)$ and $\operatorname{MDDr}(p=.003)$ groups, with the NC group scoring higher on MAAS scores than the clinical groups. However, no significant differences were found between the NC and CD groups $(p=.31)$. A significant main effect of group was also found when PHLMS-awareness scores were entered as the dependent variable $\left[F(4,228)=3.39, p=.01, \eta^{2} p=.06\right]$. Post hoc analyses revealed 
MINDFULNESS IN PYSCHIATRIC DISORDERS

significant differences between the NC and ED groups $(p=.008)$ and between NC and BPD ( $p=.03)$. No significant differences were found between the NC and CD groups $(p=.55)$ and between NC and MDDr $(p=.22)$. Given the significant between-group differences in age, sex, and educational level, preliminary analyses were conducted using these variables as co-variants to identify the significant variables to include in the final models. Neither age nor sex had a significant main effect in these preliminary ANCOVA models and thus not included in the final models. A significant main effect of education level was found only for MAAS scores.

Final models. Using MAAS scores as the dependent variable, group and educative level as the between-subjects factor and CES-D scores as a covariate, we only found a significant main effect for depressive symptoms $\left[F(1,209)=54.90, p<.001, \eta^{2} \mathrm{p}=.21\right]$. Neither the main effects of group $[F(4,209)$ $\left.=1.24, p=.29, \eta^{2} p=.02\right]$ nor educational level $\left[F(2,209)=1.28, p=.28, \eta^{2} p=.01\right]$ were significant. When PHLMS-awareness scores were entered as the dependent variable, the ANCOVA revealed no significant main effects for group $\left[F(4,215)=1.48, p=.21, \eta^{2} p=.03\right]$ or CES-D scores $[F(1,215)=3.05$, $\left.p=.82, \eta^{2} p=.01\right]$

\subsection{Between-group differences in acceptance}

Preliminary analysis. A main effect of group was found when PHLMS-acceptance scores were used in a preliminary ANCOVA $\left[F(4,229)=21.29, p<.001, \eta^{2} \mathrm{p}=.27\right]$. Post hoc analyses showed that all clinical groups scored significantly lower on acceptance than the non-clinical sample. No significant differences were observed when comparing clinical groups against each other. None of the sociodemographic variables evaluated (sex, age, educational level) were significant predictors of PHLMS-acceptance scores.

Final model. PHLMS-acceptance scores were entered as the dependent variable, group as the between-subjects factor, and CES-D scores as a covariate. In this analysis, significant main effects were found for depressive symptoms $\left[F(1,228)=17.84, p=.001, \eta^{2} p=0.07\right]$ and group $[F(4,228)=9.40, p=$ $\left..001, \eta^{2} p=.14\right]$. Post hoc analyses of PHLMS-acceptance scores revealed that all clinical groups presented significantly lower scores $(p<.05)$ than the NC group. No significant between-group differences in acceptance scores were found among the clinical groups. Table 2 shows means and standard deviations and $F$ and $p$ values of the final ANCOVA models. 
INSERT TABLE 2 HERE

\section{Discussion}

The aim of the present study was to examine differences in awareness and acceptance in different psychiatric disorders and to explore the relationship between these facets of mindfulness with depressive symptoms. Our findings suggest that deficits in the awareness facet of mindfulness are related to depressive symptoms rather than to the specific psychiatric diagnosis. By contrast, the diagnostic group and the presence of depressive symptoms were both significantly associated with acceptance scores.

When using MAAS scores as the dependent variable and CES-D scores as the co-variate, a significant effect of CES-D scores was found. This finding might indicate that impairment of attentional capacities may be more closely linked to mood alterations than to the characteristics of the specific disorder. This finding supports the MBCT framework (Teasdale et al., 2000), which suggests that acute mood alterations diminish an individual's ability to be present, suggesting that this facet of mindfulness could be mood dependent. No significant main effects were found using PHLMS-awareness scores as the dependent variable. The different results regarding the MAAS and the PHLM-awareness subscale were not surprising given that previous studies have reported weak correlations between the MAAS and the PHLMS-awareness subscale (Park et al., 2013; Tejedor et al., 2014).

As we hypothesized, all clinical groups scored significantly lower than the non-clinical sample on the acceptance domain. On the ANCOVA analysis in which PHLM-acceptance scores were used as the dependent variable, we found a significant effect of both group and CES-D scores. Although the relationship between psychopathology and the lack of acceptance-related capacities has been demonstrated in previous research (Eisenlohr-Moul et al., 2015; Kotsou et al., 2018), the present study is the first to explore this association in actual clinical populations. The relevance of acceptance has also been emphasized by Kohls et al. (2009), who found that the negative relationship between mindfulness and anxiety/depression was due primarily to the acceptance facet rather than to the awareness component of mindfulness. On the other hand, our finding is also consistent with the results reported by Kashdan et al., (2006), suggesting that acceptance is a central transdiagnostic process and that all the studied clinical groups may benefit from acceptance-oriented interventions. As expected, all of the clinical groups scored lower than the non-clinical community sample on this facet. 
MINDFULNESS IN PYSCHIATRIC DISORDERS

All mindfulness-based approaches include training in both attention and acceptance. However, some therapeutic interventions specifically developed for clinical populations - such as DBT (Linehan, 2014) or ACT (Hayes et al., 2006) — place a greater emphasis on fostering acceptance-oriented skills. In DBT (Linehan, 2014), acceptance skills are taught in order to help individuals to more effectively tolerate negative experiences (by not escalating emotions or engaging in dysregulated behaviors), whereas in the ACT framework, acceptance is considered the opposite of experiential avoidance. By enhancing acceptance skills, individuals learn to allow distressing thoughts and emotions to come and go without struggling against them (Hayes et al., 2006). The monitoring and acceptance theory (MAT; Lindsay and Creswell, 2017) emphasizes the fundamental nature of acceptance to decrease emotional distress when negative experiences arise and to promote awareness of positive experiences without attempting to "grasp" such experiences (Lindsay and Creswell, 2017). Importantly, the MAT framework highlights the synergistic effects of attention regulation and acceptance, stating that the combination of these two elements is what leads to the positive health outcomes of MBIs (Lindsay and Creswell, 2017). It is not known whether attention and acceptance skills are developed simultaneously or sequentially and this question should be investigated in future studies. In any case, the association between psychopathology and lack of acceptance, which has also been reported in other studies (Kashdan et al., 2006; Kotsou et al., 2018) provides further support for the importance of developing an acceptance-oriented approach towards experience during the early stages of mindfulness practice. Whether meditative practice is the best or only way to achieve acceptance-related capacities is not clear. In DBT, for example, acceptance is taught both through mindfulness practice - specifically in "how skills" - and through "radical acceptance skills". This latter set of skills is not based on mindfulness practice, but presents a set of psychoeducational principles developed to foster acceptance (Linehan 1993, 2014). In addition, studies exploring the effect of meditative practice on mindfulness-capacities have showed a stronger relation between mindfulness practice and other mindfulness dimensions (such as observing or decentering) than between mindfulness practice and acceptance (Franquesa et al., 2017; Soler et al., 2014). This might indicate that meditation training — which strongly relies on training attentional capacities - is not required to improve acceptanceoriented skills.

The results of this study should be interpreted in light of the study limitations, primarily that the non-clinical and clinical groups were not matched in terms of socio-demographic characteristics. As a result, there were significant differences between the groups on several socio-demographic variables. 
MINDFULNESS IN PYSCHIATRIC DISORDERS

However, it is important to emphasize that posterior analyses showed that these variables had no significant effect on mindfulness-related capacities. Another limitation is that the focus of this study was on exploring the role of depressive symptoms in present-centered awareness and acceptance and therefore we did not assess the potential role of other transdiagnostic symptoms (e.g., anxiety or impulsivity), which should be evaluated in future studies. Another limitation is the use of a two-factor model of the mindfulness construct. Our results could have been different if we had used other mindfulness measures such as the five-factor FFMQ. Indeed, had we used the FFMQ, we might have obtained a more detailed picture of the relationship between mindfulness facets, psychiatric diagnosis, and mood alterations. Nonetheless, studies that have used confirmatory analyses and exploratory structural equation modeling have suggested that the five facets of the FFMQ may be better represented by a two-factor higher order structure composed of an attentional factor and an acceptance factor (Tran et al., 2014), a finding that indicates that mindfulness is best assessed through two-factor measures. The use of self-reported instruments is another study limitation due to the inherent drawbacks of such tools. Lastly, the crosssectional study design does not allow us to make any conclusions about causality. Given that mindfulness traits fluctuate over time (Eisenlohr-Moul et al., 2015), future studies should employ ecological momentary assessments to evaluate the temporal stability of the relationship between the studied variables.

In conclusion, the findings of the present study have several interesting implications for mindfulness training in clinical groups. Our findings imply that the association between psychopathology, mindfulness, and mood alterations varies as a function of the specific mindfulness aspect (i.e., awareness or acceptance) under evaluation. Therefore, our findings do not support the conceptualization of mindfulness as a one-dimensional construct (Brown and Ryan, 2003). The current study also supports the notion of acceptance as a transdiagnostic marker of psychopathology. However, additional research is needed to disentangle whether mindfulness-based interventions exert their effects by improving disorderspecific components or by improving transdiagnostic characteristics, such as depressive symptoms. Our findings also support the use of mindfulness interventions_- particularly acceptance-related skills—-for the treatment of several psychiatric disorders. The lack of major differences between the various clinical groups in this study suggests that impairments in mindfulness-related capacities are not diagnosisspecific, and therefore interventions designed from a transdiagnostic perspective (e.g., Barlow et al., 
MINDFULNESS IN PYSCHIATRIC DISORDERS

2011) could be indicated for treating individuals who share certain clinical features, such as depressive symptoms.

\section{Acknowledgements}

The authors would like to thank Bradley Londres for his help in editing the manuscript.

\section{Funding}

This research did not receive any specific grant from funding agencies in the public, commercial, or notfor-profit sectors. 
MINDFULNESS IN PYSCHIATRIC DISORDERS

\section{References}

Arch, J.J., Craske, M.G., 2010. Laboratory stressors in clinically anxious and non-anxious individuals: the moderating role of mindfulness. Behav. Res. Ther. 48, 495-505.

Baer, R. a, Smith, G.T., Allen, K.B., 2004. Assessment of mindfulness by self-report: the Kentucky inventory of mindfulness skills. Assessment 11, 191-206.

Baer, R.A., Smith, G.T., Hopkins, J., Krietemeyer, J., Toney, L., 2006. Using self-report assessment methods to explore facets of mindfulness. Assessment 13, 27-45.

Barlow, D.H., Ellard, K.K., Fairholme, C.P., Farchione, T.J., Boisseau, C.L., Allen, L.B., et al., 2011. The unified protocol for transdiagnostic treatment of emotional disorders: Client workbook. Oxford University Press.

Bishop, S.R., Lau, M., Shapiro, S., Carlson, L., Anderson, N.D., Carmody, J.,et al., 2004. Mindfulness : A Proposed Operational Definition. Clin. Psychol. Sci. Pract. 11, 230-241.

Brown, K.W., Ryan, R.M., 2003. The benefits of being present: Mindfulness and its role in psychological well-being. J. Pers. Soc. Psychol. 84, 822-848.

Butryn, M.L., Juarascio, A., Shaw, J., Kerrigan, S.G., Clark, V., O 'planick, A., et al., 2013. Mindfulness and its relationship with eating disorders symptomatology in women receiving residential treatment. Eat. Behav. 14, 13-16.

Cardaciotto, L., Herbert, J.D., Forman, E.M., Moitra, E., Farrow, V., 2008. The assessment of presentmoment awareness and acceptance: The Philadelphia Mindfulness Scale. Assessment 15, 204-223.

Eisenlohr-Moul, T. A., Peters, J.R., Chamberlain, K.D., Rodriguez, M. A., 2015. Weekly Fluctuations in Nonjudging Predict Borderline Personality Disorder Feature Expression in Women. J. Psychopathol. Behav. Assess. 38(1),149-157.

Eisenlohr-Moul, T., Peters, J.R., Chamberlain, K.D., Rodriguez, M., 2016. Weekly fluctuations in nonjudging predict borderline personality disorder feature expression in women. J. Psychopathol. Behav. Assess. 38, 149-157.

Elices, M., Pascual, J.C., Carmona, C., Martín-Blanco, A., Feliu-Soler, A., Ruiz, E., et al., 2015. Exploring the relation between childhood trauma, temperamental traits and mindfulness in borderline personality disorder. BMC Psychiatry 15, 180-187.

Elices, M., Pascual, J.C., Portella, M.J., Feliu-Soler, A., Martín-Blanco, A., Carmona, C., Soler, J., 2016. Impact of Mindfulness Training on Borderline Personality Disorder: A Randomized Trial. 
MINDFULNESS IN PYSCHIATRIC DISORDERS

Mindfulness. 7, 584-595.

First, M., Spitzer, R.L., Gibbon, M., Williams, J.B., 1999. Structured Clinical Interview for DSM-IV Axis I Disorders. Masson, Barcelona, Spain.

Franquesa, A., Cebolla, A., García-Campayo, J., Demarzo, M., Elices, M., Pascual, et al., 2017.

Meditation Practice Is Associated with a Values-Oriented Life: the Mediating Role of Decentering and Mindfulness. Mindfulness. 8, 1259-1268.

Gibbon, M., Spitzer, R.L., 1997. User's guide for the structured clinical interview for DSM-IV axis II personality disorders: SCID-II. American Psychiatric Publishing.

Hayes, S.C., Luoma, J.B., Bond, F.W., Masuda, A., Lillis, J., 2006. Acceptance and Commitment Therapy: Model, processes and outcomes. Beh.Res.Ther. 44(1), 1-25.

Hayes, S.C., Strosahl, K., Wilson, K.G., 1999. Acceptance and commitment therapy: Understanding and treating human suffering. Guilford Press, New York.

Kabat-Zinn, J., 1990. Full catastrophe living: Using the wisdom of your body and mind to face stress, pain, and illness. Delacorte, New York.

Kashdan, T., Barrios, V., Forsyth, J., Steger, M., 2006. Experiential avoidance as a generalized psychological vulnerability: Comparisons with coping and emotion regulation strategies. Behav. Res. Ther. 44, 1301-1320.

Khoury, B., Lecomte, T., Fortin, G., Masse, M., Therien, P., Bouchard, V., et al., 2013. Mindfulnessbased therapy: a comprehensive meta-analysis. Clin. Psychol. Rev. 33(6), 763-71.

Khoury, B., Sharma, M., Rush, S.E., Fournier, C., 2015. Mindfulness-based stress reduction for healthy individuals: A meta-analysis. J. Psychosom. Res. 78, 519-528.

Kohls, N., Sauer, S., Walach, M.H., 2009. Facets of Mindfulness - Results of an online study investigating the Freiburg Mindfulness Inventory. Pers. Individ. Dif. 46, 224-230.

Kotsou, I., Leys, C., Fossion, P., 2018. Acceptance alone is a better predictor of psychopathology and well-being than emotional competence, emotion regulation and mindfulness. J. Affect. Disord. 226, 142-145.

Krantz, L.H., McMain, S., Kuo, J.R., 2018. The unique contribution of acceptance without judgment in predicting nonsuicidal self-injury after 20-weeks of dialectical behaviour therapy group skills training. Beh.Res.Ther. 104, 44-50.

Lavender, J.M., Gratz, K.L., Tull, M.T., 2011. Exploring the relationship between facets of mindfulness 
MINDFULNESS IN PYSCHIATRIC DISORDERS

and eating pathology in women. Cogn. Behav. Ther. 40, 174-182.

Lindsay, E.K., Creswell, J.D., 2017. Mechanisms of mindfulness training: Monitor and Acceptance Theory (MAT). Clin. Psychol. Rev. 51, 48-59.

Linehan, M.M., 1993. Cognitive-behavioral treatment of borderline personality disorder. Guilford Press, New York.

Linehan, M., 2014. DBT. Skills Training Manual. Guilford Publications, New York.

Peckham, A.D., McHugh, R.K., Otto, M.W., 2010. A meta-analysis of the magnitude of biased attention in depression. Depress. Anxiety 27, 1135-1142.

Radloff, L.S., 1977. The CES-D Scale: A Self-Report Depression Scale for Research in the General Population. Appl. Psychol. Meas. 1, 385-401.

Shorey, R.C., Brasfield, H., Anderson, S., Stuart, G.L., 2013. Mindfulness deficits in a sample of substance abuse treatment seeking adults: a descriptive investigation. J. Subst. Use. 19(1-2), 194 198.

Soler, J., Domínguez-Clavé, E., García-Rizo, C., Vega, D., Elices, M., Martín-Blanco, A., et al., 2016. Validación de la versión española del McLean Screening Instrument for Borderline Personality Disorder. Rev. Psiquiatr. Salud Ment.9,195-202.

Soler, J., Franquesa, A., Feliu-Soler, A., Cebolla, A., Garcia-Campayo, J., Tejedor, R., et al., 2014. Assesing decentering: Validation, psychometric properities and clinical usefulness of the Experiences Questionnaire in a Spanish sample. Behav. Ther.45, 863-871.

Soler, J., Pérez-Sola, V., Puigdemont, D., Pérez-Blanco, J., Figueres, M., Alvarez, E., 1997. Estudio de validación del Center for Epidemiologic Studies-Depression (CES-D) en una población española de pacientes con trastornos afectivos. Actas españolas Psiquiatr. 25, 243-249.

Soler, J., Tejedor, R., Feliu-Soler, A., Pascual, J.C., Cebolla, A., Soriano, J., et al., 2012. Psychometric proprieties of Spanish version of Mindful Attention Awareness Scale (MAAS). Actas Esp. Psiquiatr. 40, 19-26.

Teasdale, J.D., Segal, Z. V, Williams, J.M., Ridgeway, V. A, Soulsby, J.M., et al., 2000. Prevention of relapse/recurrence in major depression by mindfulness-based cognitive therapy. J. Consult. Clin. Psychol. 68, 615-623.

Teasdale, J.D., Segal, Z., Williams, M.., 1995. How does cognitive therapy prevent depressive relapse and why should attentional control (mindfulness) training help? Behav. Res. Ther. 33, 25-39. 
MINDFULNESS IN PYSCHIATRIC DISORDERS

Tejedor, R., Feliu-Soler, A., Pascual, J.C., Cebolla, A., Portella, M.J., Trujols, J., et al., 2014. Propiedades psicométricas de la versión española de la Philadelphia Mindfulness Scale. Rev Psiquiatr Salud Ment. 7, 157-165.

Tran, U.S., Cebolla, A., Glück, T.M., Soler, J., Garcia-Campayo, J., von Moy, T., 2014. The serenity of the meditating mind: a cross-cultural psychometric study on a two-factor higher order structure of mindfulness, its effects, and mechanisms related to mental health among experienced meditators. PLoS One. 9(10), e110192.

Wupperman, P., Neumann, C.S., Axelrod, S.R., 2008. Do deficits in mindfulness underlie borderline personality features and core difficulties? J. Pers. Disord. 22, 466-82.

Zanarini, M.C., Vujanovic, a A., Parachini, E. a, Boulanger, J.L., Frankenburg, F.R., Hennen, J., 2003. A screening measure for BPD: the McLean Screening Instrument for Borderline Personality Disorder (MSI-BPD). J. Pers. Disord. 17, 568-73. 
MINDFULNESS IN PYSCHIATRIC DISORDERS 


\section{Table 1}

Socio-demographic and clinical characteristics by group

\begin{tabular}{|c|c|c|c|c|c|c|c|c|}
\hline & & & Groups & & & & & \\
\hline & $\begin{array}{c}\mathrm{NC} \\
(n=76)\end{array}$ & $\begin{array}{c}\text { CD } \\
(n=43)\end{array}$ & $\begin{array}{c}\text { ED } \\
(n=43)\end{array}$ & $\begin{array}{c}\text { BPD } \\
(n=45)\end{array}$ & $\begin{array}{c}\text { MDDr } \\
(n=39)\end{array}$ & $\boldsymbol{F}$ & $X^{2}$ & $p$ \\
\hline Gender (\% of women) & 71.1 & 18.6 & 93.0 & 91.1 & 79.5 & & 73.89 & .001 \\
\hline Age, M (SD) & $33.30(11.90)$ & $35.10(7.88)$ & $30.12(11.48)$ & $29.03(7.92)$ & $48.32(13.33)$ & 20.27 & & .001 \\
\hline \multicolumn{9}{|l|}{ Education level (\%) } \\
\hline - Less than high school & 0.0 & 73.2 & 9.3 & 36.4 & 27.0 & & 145.98 & .001 \\
\hline - High school graduate & 15.8 & 22.0 & 58.1 & 39.4 & 27.0 & & & \\
\hline - University graduate & 84.2 & 4.9 & 32.6 & 21.2 & 45.9 & & & \\
\hline Employment situation (\%) & & & & & & & 124.69 & .001 \\
\hline - Employed & 75.0 & 36.6 & 23.8 & 17.6 & 35.1 & & & \\
\hline - Unemployed & 19.7 & 2.4 & 38.1 & 32.3 & 21.6 & & & \\
\hline - Medical leave & 5.3 & 61.0 & 38.1 & 50.0 & 37.8 & & & \\
\hline Marital Status (\%) & & & & & & & 58.88 & .001 \\
\hline - Single & 36.8 & 55.0 & 67.4 & 51.5 & 11.1 & & & \\
\hline - Married/Cohabiting & 60.5 & 22.5 & 23.8 & 30.3 & 72.2 & & & \\
\hline - Divorced/Widowed & 2.6 & 22.5 & 7.0 & 18.2 & 16.7 & & & \\
\hline CES-D, M (SD) & $6.57(6.02)$ & $19.67(11.51)$ & $28.40(12.14)$ & $37.80(13.42)$ & $22.92(16.01)$ & 57.79 & & .001 \\
\hline
\end{tabular}

Note. $\mathrm{NC}=$ Non-clinical $. \mathrm{CD}=$ Cocaine Dependence. $\mathrm{ED}=$ Eating Disorder. $\mathrm{BPD}=$ Borderline Personality Disorder. $\mathrm{MDDr}=\mathrm{Major}$ Depressive Disorder in remission. CESD-D = Centre for Epidemiologic Studies Depression Scale. $\mathrm{M}=$ Mean. SD = Standard Deviation. 


\section{Table 2}

MASS and PHLMS scores by diagnostic group. F and p values from analysis of co-variance (final models)

\begin{tabular}{|c|c|c|c|c|c|c|c|c|c|c|c|c|c|c|c|c|}
\hline & & & & & & & & & & & \multicolumn{6}{|c|}{ ANCOVA } \\
\hline & \multicolumn{2}{|c|}{$\mathrm{NC}$} & \multicolumn{2}{|c|}{ CD } & \multicolumn{2}{|c|}{ ED } & \multicolumn{2}{|c|}{ BPD } & \multicolumn{2}{|c|}{ MDDr } & \multicolumn{2}{|c|}{ Group } & \multicolumn{2}{|c|}{$\begin{array}{c}\text { Depressive } \\
\text { Symptoms } \\
(\text { CES-D) } \\
\end{array}$} & \multicolumn{2}{|c|}{ Education level } \\
\hline & $M$ & $(S D)$ & $M$ & $(S D)$ & $M$ & $(S D)$ & $M$ & $(S D)$ & $M$ & $(S D)$ & $F$ & $p$ & $F$ & $p$ & $F$ & $p$ \\
\hline MAAS $^{\mathbf{a}}$ & 4.38 & $(0.11)$ & 4.21 & $(0.14)$ & 3.88 & $(0.14)$ & 3.25 & $(0.16)$ & 3.84 & $(0.15)$ & 1.24 & .29 & 54.90 & .001 & 1.28 & .28 \\
\hline $\begin{array}{l}\text { PHLMS- } \\
\text { awareness }^{\text {b }}\end{array}$ & 33.80 & $(5.06)$ & 34.39 & $(5.34)$ & 30.80 & (5.79) & 31.25 & (5.96) & 32.37 & $(5.97)$ & 1.48 & .21 & 3.05 & .82 & & \\
\hline $\begin{array}{l}\text { PHLMS- } \\
\text { acceptance }^{c^{*}}\end{array}$ & 32.76 & $(6.45)$ & 24.65 & $(7.26)$ & 23.65 & $(6.04)$ & 25.53 & (5.64) & 24.41 & $(6.51)$ & 9.40 & .001 & 17.84 & .001 & & \\
\hline
\end{tabular}

Note $. \mathrm{NC}=$ Non-clinical. $\mathrm{CD}=$ Cocaine dependence $. \mathrm{ED}=$ Eating disorder. $\mathrm{BPD}=$ Borderline Personality Disorder. $\mathrm{MDDr}=$ Major Depressive Disorder in remission.

$M=$ Mean. $\mathrm{SD}=$ Standard Deviation. MAAS = Mindfulness Attention Awareness Scale. PHLMS = Philadelphia Mindfulness Scale. CESD-D = Centre for Epidemiologic Studies Depression Scale.

${ }^{a}$ MAAS analyses are based on the following: $\mathrm{NC}=75, \mathrm{CD}=41, \mathrm{ED}=43, \mathrm{BPD}=28, \mathrm{MDDr}=37$. Covariates entered: CES-D scores and educational level.

${ }^{\mathrm{b}} \mathrm{PHLMS}$-acceptance analyses are based on the following: $\mathrm{NC}=72, \mathrm{CD}=41, \mathrm{ED}=42, \mathrm{BPD}=30, \mathrm{MDDr}=31$. Covariate entered: CES-D scores.

'PHLMS-awareness analyses are based on the following: $\mathrm{NC}=72, \mathrm{CD}=41, \mathrm{ED}=40, \mathrm{BPD}=30, \mathrm{MDDr}=32$. Covariate entered: CES-D scores.

*The non-clinical sample had significantly higher acceptance scores than the clinical groups. 